Climate Dynamics (1993) 9:71-80

\title{
On the momentum forcing of a large-scale sea-ice model
}

\author{
Achim Stössel and Martin Claussen \\ Max-Planck-Institut für Meteorologie, Bundesstrasse 55, D-20146 Hamburg, Germany
}

Received 2 November 1992 / Accepted 26 February 1993

\begin{abstract}
A large-scale sea-ice - oceanic mixed-layer model for the Southern Ocean is forced with daily atmospheric fields from operational numerical weather prediction analyses. The strength of the atmospheric forcing is modified considering atmospheric surfacelayer physics, which is itself directly dependent on the instantaneous sea-ice condition provided by the sea-ice model. In earlier applications, the atmospheric drag on sea ice was computed from the local momentum transfer over ice. In the present study, this is replaced by a large-scale momentum flux, which is characterized by a large-scale stability function and a large-scale roughness length. The large-scale roughness length depends on the local skin drags and on the form drag, where the latter is given as a function of the ice-plus-snow freeboard and the ice concentration, both provided by the sea-ice model. The thermodynamic part of the calculation is given by the local fluxes, which depend on the local stability of the atmospheric surface layer. This, physically more reasonable, description of the largescale dynamic forcing generally leads to an increase of the momentum transfer via an increase of the roughness length and a decrease of the stability in the atmospheric surface layer. Finally, this yields improved model results, especially in terms of a more dynamic pattern of the ice-thickness distribution.
\end{abstract}

\section{Introduction}

Scientists engaged in coupled ocean - atmosphere general circulation model (GCM) experiments have generally recognized that the simulation results in high and mid-latitudes are highly dependent on the treatment of the sea-ice component (Meehl and Washington 1990;

This paper was presented at the Second International Conference on Modelling of Global Climate Variability, held in Hamburg 7-11 September 1992 under the auspices of the Max Planck Institute for Meteorology. Guest Editor for these papers is L. Dümenil
Manabe et al. 1992; Cubasch et al. 1992). One problem is related to the physical description of the sea-ice component itself, which in coupled GCMs is usually rather crude in comparison to sea-ice models used for regional polar studies. Specifically, certain first-order effects that occur e.g. due to the consideration of an ice rheology for the ice dynamics or a comprehensive heatbudget calculation including the insulation effect of snow (Stössel et al. 1990), are usually not properly determined.

The other crucial part of the sea-ice treatment is the proper determination of the fluxes between the atmosphere, sea-ice and ocean components. In order to ensure this, it appears to be necessary to resolve more physical processes within the transition zones from one component to the other, i.e. to account for boundarylayer processes including sub-grid scale features due to surface heterogeneities.

This is a problem which ought to be addressed also in forced experiments. Questions concerning interactive and boundary-layer processes together with the specification of the appropriate forcing (coupling) interface are inherent and decisive for forced simulations, too.

In sea-ice regions the dominant factors controlling the strength of the forcing or coupling between atmosphere and sea ice originate from the extremely heterogeneous horizontal distribution of static stability within the atmospheric boundary layer, and the variations in surface roughness due to variable ice and snow thicknesses, deformations in the form of pressure ridges and variable ice coverage. These aspects are referred to in Stössel (1992) and Claussen (1991a).

Another aspect not very well considered so far is related to the large-scale application of the sea-ice component. Since sea-ice simulations on GCM scales are far from resolving ice floes, sea ice is treated as a continuum. However, options are provided to present a grid cell partially ice covered (expressed by the ice concentration or ice compactness), dividing the grid cell into two regions with totally different characteristics of the respective (local) atmospheric boundary 
layers. Furthermore, the local boundary layers merge to a mixture of their characteristics beyond a certain height above the surface (the blending height) depending on the specific distribution of the ice-covered and ice-free part within a grid cell.

In earlier sea-ice simulations (Stössel 1992) the forcing level was established at a certain height above the surface in order to introduce modifications of the forcing based on atmospheric surface-layer (ASL) physics. Specifying the atmospheric forcing above the blending height suggests the boundary-layer modifications of the dynamic forcing to be determined by "large-scale" or "effective" boundary-layer quantities. These are calculated as functions of the ice concentration, ice freeboard, snow thickness and the local surface-layer quantities, representing the main issue of this study.

After some basic comments on the applied models and forcing fields in Section 2, a detailed description of the large-scale modifications to the earlier atmospheric surface-layer formulation is given in Section 3. The impact of these modifications on the results are presented in Section 4, primarily as differences from studies with local derivation of the momentum transfer.

\section{Basic models and forcing fields}

The basic model consists of a dynamic-thermodynamic sea-ice model coupled to an oceanic mixed-layer model, both described in detail in Lemke et al. (1990). Snow condition is described according to Owens and Lemke (1990). Atmospheric boundary-layer parameterizations were introduced by Stössel (1992). The earlier literature is referred to for the model formulations, since they are comprehensive and not critical for the present study.

As in the earlier studies, the region of interest is the entire Southern Ocean. Following Stössel (1992), the model grid is spherical with $\Delta \varphi=2.5^{\circ}$ and $\Delta \lambda=5^{\circ}$, the time step is one day and the integration time 6 years to achieve cyclostationarity with respect to the ice volume. The main atmospheric forcing variables consist of real-time daily mean temperature, humidity and winds at the $1000 \mathrm{hPa}$ level from the global analyses of the European Centre for Medium Range Weather Forecasts (ECMWF) (Trenberth and Olson 1988). The surface pressure is derived from variables of the same data set. The reasons for using these essentially model generated data and the reconstruction of the actual forcing level are described in Stössel (1992). As in the earlier studies, the final year of integration is determined by the real-time forcing of the year 1986. All other forcing variables are derived from climatologies, also being equivalent to the earlier specifications (see Stössel et al. 1990).

The equations of interest for the present study are those of the atmospheric surface-layer parameterization and those related to the heterogeneous surface of a model grid cell. In the earlier applications it was assumed that the boundary layers develop separately (locally) over the ice-free and the ice-covered part of a grid cell. In the present study, this scheme is retained to calculate the local heat budgets, which largely determine the ice growth rates. Specifically, it is assumed that the turbulent heat fluxes are governed by the local stabilities of the respective ASLs. The motivation for this approach is given in Stössel (1992) and is summarized in the following.

Independent of the particular atmospheric forcing used, there is only one forcing variable (e.g. air temperature) per grid cell available. This actually ought to be a blend of data sampled over the instantaneous (heterogeneous) sea-ice distribution in the grid cell area or, in case of an atmospheric GCM (AGCM) product, a result depending on the instantaneous boundary conditions of the AGCM. By raising the forcing level to the top of the ASL, the requirement for local atmospheric surface forcing variables $(2 \mathrm{~m}$ or $10 \mathrm{~m}$ quantities) is relaxed, the local forcing being determined via the local ASL properties.

The formulas describing the atmospheric surface layers were derived from Louis (1979). They are based on the Monin-Obukhov similarity theory, the MoninObukhov length being replaced by the Richardson number for the lowest atmospheric layer. The local Richardson numbers over the ice-covered $(i)$ and the ice-free $(o)$ parts yield:

$R i_{i, o}=g z_{a}\left(\theta_{a}-\theta_{i, o}+0.61 \theta_{a}\left(q-q_{i, o}\right)\right) /\left(\theta_{a}\left|\vec{V}_{a}\right|^{2}\right)$,

where: $g$ : acceleration due to gravity,

$z_{a}$ : height of the lowest atmosphere layer (=forcing level),

$\theta_{a}:$ potential temperature of the lowest atmosphere layer,

$\theta_{i}$ : potential temperature at ice-covered surface $\left(=T_{i}\right)$,

$\theta_{o}:$ potential temperature at ice-free surface $\left(=T_{o}\right)$,

$T_{i, o}:$ local surface temperatures,

$q$ : specific humidity of lowest atmosphere layer,

$q_{i, o}:$ specific humidities at the local surfaces,

$\vec{V}_{a}$ : wind in the lowest atmosphere layer.

Here, the surface variables are determined by the local surface properties, e.g. $T_{i}$ by the ice- or snow-surface temperature in the ice-covered part and $T_{o}$ by the seasurface temperature [=oceanic mixed-layer temperature $T_{o 1}$ in Stössel (1992)] in the ice-free part (=freezing point, when ice occurs within a grid cell).

In the present application, $z_{a}=30 \mathrm{~m}$, unless the surface pressure exceeds $\approx 1004 \mathrm{hPa}$, i.e. the $1000 \mathrm{hPa}$ level lies above $30 \mathrm{~m}$. In this case, $z_{a}$ increases together with the $1000 \mathrm{hPa}$ level (Stössel 1992).

The local friction velocities $\left(u_{* i, o}\right)$ are defined from the vertical eddy fluxes of momentum:

$\left(\overline{w^{\prime} u^{\prime}}\right)_{i, o} \equiv u_{* i, o}^{2}=\left(\frac{\kappa}{\ln \left(z_{a} / z_{0 i, o}\right)}\left|\vec{V}_{a}\right|\right)^{2} F_{M i, o}$,

where $\kappa: \quad$ von Karman constant

$z_{0 i, o}:$ local roughness lengths

$F_{M i, a}:$ stability functions for local momentum fluxes. 
Following Stössel (1992), the local roughness length over ice is specified as $z_{0 i}=1 \cdot 10^{-3} \mathrm{~m}$. The roughness over the ice-free part is determined by the local wind-generated waves, implicitly formulated by $z_{0 \circ}$ $=C u_{* o}^{2} / g$, where $C=0.032$ is the Charnock constant.

The corresponding scaling temperatures $\left(\theta_{* i, o}\right)$ are assumed to be related to the vertical eddy fluxes of sensible heat:

$$
\begin{aligned}
-\left(\overline{w^{\prime} \theta^{\prime}}\right)_{i, o} & \equiv\left(u_{*} \theta_{*}\right)_{i, o}=-\frac{Q_{S E i, o}}{\rho_{a} c_{p a}} \\
& =\frac{\kappa^{2}\left|\vec{V}_{a}\right|\left(\theta_{a}-\theta_{i, o}\right) F_{H i, o}}{0.74 \ln ^{2}\left(z_{a} / z_{0 i, o}\right)},
\end{aligned}
$$

where $Q_{S E i, o}:$ local sensible heat fluxes,

$\rho_{a}: \quad$ air density,

$c_{p a}: \quad$ specific heat of air at constant pressure,

$F_{H i, o}:$ stability functions for local turbulent heat fluxes.

The (local) latent heat fluxes $\left(Q_{L A i, o}\right)$ are given by analogous formulas with $q$ and $q_{i, o}$.

After a slight modification according to Claussen (1991a), the stability functions combine as follows:

$$
\begin{aligned}
F_{M, H} & =\left(\frac{1}{(1+2 \cdot 4.7 R i)^{2}}\right) \delta_{j k} \\
& +\left(1-\frac{9.4 R i}{1+c_{M, H}|R i|^{0.5}}\right)\left(1-\delta_{j k}\right),
\end{aligned}
$$

where $\delta$ is the Kronecker symbol and $j=k$, if $R i \geq 0$, with

$c_{M, H}=(7.4,5.3)\left(\frac{\kappa}{\ln \left(z_{a} / z_{0}\right)}\right)^{2} 9.4 \sqrt{\frac{z_{a}}{z_{0}}}$.

For neutral and convective stratifications these formulas follow from analytical adaptations to the functions of Businger et al. (1971). In the case of highly stable stratifications, the expression for $F_{M, H}$ follows from an asymptotic approach toward zero for increasing Richardson numbers (Louis 1979).

\section{Modifications for large-scale applications}

In earlier applications (Stössel 1992), the momentum transfer from the atmosphere to the ice was determined by local properties. However, observations and simulations (e.g. Hanssen-Bauer and Gjessing 1988; Andreas et al. 1984; Overland and Davidson 1992; Claussen 1991a; Simmonds and Budd 1991; Worby and Allison 1991; Stössel 1992) suggest this momentum transfer to be determined by aggregated, grid-cell averaged quantities. For large-scale modelling sub-grid scale effects due to heterogeneous compositions at the surface may be considered by weighting the local properties linearly with the surface composition. Observations, however, imply that certain surface features, e.g. leads in the case of sea ice, may dominate the largescale characteristics, even when their fraction is rather small (Claussen 1991b). This non-linear behaviour is accounted for in the present study by a blend of the local ASL properties. The translation of the sub-grid scale properties to the large-scale quantities is described in the following.

First, it is necessary to introduce a measure for the surface roughness in terms of an "effective" or "largescale" roughness length. In sea-ice areas with ice compactness lower than $100 \%$, the effective roughness length is determined by the freeboard of the (level) ice floes (including snow), by the size of the floes, by pressure ridges, by the local skin frictions and by the seasurface roughness of the ice-free parts (waves). by

The (effective) ice (plus snow) freeboard is given

$h_{f}=\left(h_{i}\left(1-\rho_{i} / \rho_{w}\right)+h_{s}\left(1-\rho_{s} / \rho_{w}\right)\right) / N_{i}$,

where $\rho_{i}$ : density of sea ice,

$\rho_{w}$ : density of water,

$\rho_{s}$ : density of snow,

$h_{i}$ : mean (grid cell averaged) ice thickness,

$h_{s}$ : mean snow thickness,

$N_{i}$ : ice coverage per grid cell.

In order to avoid exaggerated freeboards at locations where snow has accumulated to a few metres, a snow to ice conversion is introduced closely following Leppäranta (1983) and Stössel (1985). Instead of creating a new aggregate in the form of snow ice, however, snow is directly converted into ice whenever the weight of the snow exceeds the buoyancy of the ice-plus-snow column to an extent that the snow submerges below the water line. The draught of the ice-plus-snow column is given by:

$h_{d}=\left(h_{s} \rho_{s} / \rho_{w}+h_{i} \rho_{i} / \rho_{w}\right) / N_{i}$.

Whenever $h_{d}>\tilde{h}_{i}$ (where $\tilde{h}_{i}$ is the effective ice thickness defined as $\left.\tilde{h}_{i}=h_{i} / N_{i}\right), \tilde{h}_{i}$ is set equal to $h_{d}$ and the (effective) snow thickness $\left(\tilde{h}_{s}=h_{s} / N_{i}\right)$ is reduced by

$\Delta \tilde{h}_{s}=\left(\tilde{h}_{i}-h_{d}\right) \rho_{i} / \rho_{s}$.

Additionally, it is necessary to know the horizontal scale of roughness variations, $\hat{L}$. Over sea ice $\hat{L}=L_{i}+L_{o}$, where $L_{i}$ is the floe size and $L_{o}$ the lead size, both considered parallel to the upwind direction. Since these quantities are not provided by the sea-ice model, the average floe size is assumed to be given as a function of the effective ice thickness, e.g. $L_{i}=\tilde{h}_{i} \cdot 100$. As will be shown later, this estimation can be related to the "relative length $\beta$ " $\left(\beta=L_{i} / h_{f}\right)$ introduced by Hanssen-Bauer and Gjessing (1988), hereinafter referred to as $\mathrm{HG}$.

The sea-ice model does not provide any information about the height and frequency of pressure ridges. Thus, its direct roughness contributions are neglected. However, there is some indirect compensation due to the addition of snow, which in the present formulation is added as a rigid body on top of the ice (Eq. 5). Since snow usually is smoothed at the windward edges, its contribution to the surface roughness is thus somewhat overestimated.

After the surface roughness conditions have been introduced, the derivation of the large-scale ASL quantities is described in the following. 
In order to express the effective wind stress (also called total wind drag) $\tilde{\tau}_{a}$ over a heterogeneous area of sea ice, the drag-partition theory is used (Arya 1975):

$\tilde{\tau}_{a}=\left\langle\tau_{S}\right\rangle+\tau_{F}$,

where $\left\langle\tau_{S}\right\rangle$ : mean skin drag $\left(=N_{i} \tau_{S i}+\left(1-N_{i}\right) \tau_{S o}\right)$,

$\tau_{S i}:$ local skin drag over ice-covered part,

$\tau_{S o}:$ local (undisturbed) skin drag over ice-free part,

$\tau_{F}$ : form drag due to floe edges.

A detailed discussion of the drag partition theory is found in Marshall (1971) together with its evaluation in wind-tunnel experiments.

In turbulent flow over rough surfaces, the division between skin and form drag is somewhat arbitrary. In the present application, the skin drag is considered to be that portion of the drag which is associated with roughness elements with dimensions of the order of a few centimetres or less. The effect of these small elements on the surface-layer flow is determined by the local roughness lengths of ice $\left(z_{0 i}\right)$ and of water $\left(z_{0 o}\right)$, respectively. The influence of larger roughness elements are represented by the form drag, which is part of the parameterization of the effective roughness length.

In accordance with Arya (1975), it is assumed that wakes, which originate at downwind obstacles, blend at a height $z_{b}$ such that for heights $z_{a} \ll z_{b}$ the flow is in equilibrium with the local surface, whereas for $z_{a} \gg z_{b}$ single wakes are presumed not to be identified individually, i.e. the flow just 'feels' a rougher surface. For heights $z_{a} \ll z_{b}$, and not too close to the obstacles, the wind profile can be parameterized by the local roughness lengths and local friction velocities. For $z_{a} \gg z_{b}$, on the other hand, the wind profile is a function of an effective roughness length $\tilde{z}_{0}$ and an effective friction velocity $\tilde{u}_{*}$. At the blending height, $z_{b}$, both conditions are approximately met. Thus, the ratio of the total aerodynamic drag to the (undisturbed) skin drag over open water yields

$\frac{\tilde{\tau}_{a}}{\tau_{S o}}=\left(\frac{\ln \left(z_{b} / z_{0 o}\right)}{\ln \left(z_{b} / \tilde{z}_{0}\right)}\right)^{2}$,

so that for the effective roughness length $\tilde{z}_{0}$ :

$\ln \left(\tilde{z}_{0} / z_{0 o}\right)=\ln \left(z_{b} / z_{0 o}\right)\left(1-\left(\tilde{\tau}_{a} / \tau_{S o}\right)^{-0.5}\right)$.

In order to compute the effective roughness length, the mean skin drag $\left(\left\langle\tau_{S}\right\rangle\right)$ and the form drag $\left(\tau_{F}\right)$ have to be known. The form drag is parameterized in the conventional manner (e.g. Marshall 1971) by assuming that $\tau_{F}$ is proportional to a 'roughness-element' drag coefficient $c_{d}$, the lateral cover $h_{f} / \hat{L}$ of roughness elements, and the square of mean velocity at a height $h_{f} f e$, i.e. $\left|\vec{V}_{a}\left(h_{f} / e\right)\right|^{2}=\left(\tau_{S o} / \rho_{a}\right)\left(\ln \left(h_{f} f\left(e z_{0 o}\right)\right) / \kappa\right)^{2}$, where $e=\exp (1)$. In addition, it is assumed that sheltering effects at floe edges can be considered by introducing a factor $s=\left(1-\exp \left(-0.18 L_{o} / h_{f}\right)\right.$ according to $\mathrm{HG}$. Hence, the ratio of the form drag to the open-water skin drag can be written as
$\frac{\tau_{F}}{\tau_{S o}}=\frac{c_{d} h_{f}}{2 \hat{L}}\left(\frac{s}{\kappa} \ln \left(h_{f}\left(e z_{0 o}\right)\right)\right)^{2}$,

where $c_{d}$ is set equal to 1 , following HG.

With respect to the estimation $L_{i}=\tilde{h}_{i} \cdot 100$ given earlier, the approximation $h_{f} \approx 0.1 \cdot \tilde{h}_{i}$ in case of zero snow cover yields $\beta \approx 1000$. Accounting for snow, however, with $h_{s}$ being on average some $10 \%$ of $h_{i}, \beta$ yields a value of approximately 500 . Quantifying this value in terms of $\tau_{F} / \tau_{S o}$ as a function of ice compactness (HG), the maximum form drag is achieved at $N_{i} \approx 90 \%$. This value is in good agreement with observations across the Southern Ocean ice edge (Andreas et al. 1984).

The ratio of the mean skin drag to the open-water skin drag is estimated by assuming that the large-scale turbulent fluxes merge at sufficiently large heights above the ground. It is suggested that this height is roughly as large as the blending height $z_{b}$. Furthermore, attenuation of skin drag on narrow leads due to wind reduction upwind and downwind of ice-floe edges is neglected. It was shown by HG that this effect is small for $\beta \geq 500$ (its sensitivity will be evaluated in Sect. 4.3). Hence

$\frac{\left\langle\tau_{S}\right\rangle}{\tau_{S o}}=\left(\frac{\ln \left(z_{b} / z_{0 o}\right)}{\ln \left(z_{b} /\left(z_{0}\right)\right)}\right)^{2}$,

where $\left\langle z_{0}\right\rangle$ is the aggregated roughness length of $z_{0 i}$ and $z_{0 o}$. As shown in Claussen (1991a), $\left\langle z_{0}\right\rangle$ can be derived from

$\ln \left\langle z_{0}\right\rangle=N_{i} \ln z_{0 i}+\left(1-N_{i}\right) \ln z_{0 o}$,

providing a reasonable estimate.

For estimating the blending height it is assumed that the dispersion of wakes generated at the ice-floe edges is governed by local advection, i.e. basically by horizontal advection with the mean flow and vertical turbulent diffusion. From the theory of local advection it follows that

$\ln \left(z_{b} /\left\langle z_{0}\right\rangle\right) z_{b} / \hat{L}=$ const.,

where const. $\cong 0.35$ according to Claussen (1991b). A simple, but convenient approximation to Eq. (14a) is

$z_{b}=\left\langle z_{0}\right\rangle^{0.2}(\hat{L} / 2)^{0.8}$.

Actually it turns out that a precise evaluation of $z_{b}$ is not necessary, because the large-scale momentum flux does not vary significantly with $z_{b}$ for the range of $\left\langle z_{0}\right\rangle$ and $\hat{L}$ considered here.

With respect to the turbulent heat fluxes, empirical data discussed in Beljaars (1982) and Beljaars and Holtslag (1991) support the conjecture that over a terrain with steep roughness elements, they are not directly affected by the form drag of such elements. Therefore, Claussen (1992) suggests evaluating only large-scale momentum fluxes from the large-scale roughness length, whereas turbulent heat fluxes should be estimated from local roughness lengths. Thus, it is consistent with Stössel (1992) to compute the turbulent heat fluxes locally over each surface type, with the forcing being specified at approximately the level of the blending height (Claussen 1991b). Since the local 
roughness lengths of ice and water do not differ strongly, computation of heat fluxes at any level $z_{a}$ within the surface layer seems to be a fair approximation, provided that $z_{a}$ and $z_{b}$ are of the same order of magnitude (Claussen 1991a). Therefore, the turbulent heat fluxes are calculated locally using Eq. (3).

While the local momentum fluxes (due to skin drag only) are obtained in a similar way according to Eq. (2), the large-scale momentum flux is additionally to the large-scale roughness length modified by a largescale stability. Its effect on the large-scale friction velocity is given by:

$\tilde{u}_{*}^{2}=\left(\frac{\kappa}{\ln \left(z_{a} / \tilde{z}_{0}\right)}\left|\vec{V}_{a}\right|\right)^{2} \tilde{F}_{M}(\tilde{R} i)$,

where $\tilde{F}_{M}$ : large-scale stability function, $\tilde{R} i$ : large-scale Richardson number.

This friction velocity determines the effective drag coefficient $\left(\tilde{C}_{d}=\left(\tilde{u}_{*} /\left|\vec{V}_{a}\right|\right)^{2}\right)$, which finally modifies the stress between atmosphere and sea ice $\left(\tilde{\tau}_{a}\right)$.

The large-scale stability function is determined according to Eq. (4), where the local Richardson number is substituted by $\widetilde{R} i$. The latter is evaluated by assuming that the large-scale stability is not directly affected by the form drag of obstacles (as mentioned for the heat fluxes). Hence, $\tilde{F}_{M}$, and thus $\tilde{R} i$, are approximated from the averaged stresses ignoring form drag, i.e.:

$\tilde{F}_{M}=\frac{\left\langle u_{*}^{2}\right\rangle}{C_{d}\left(\left\langle z_{0}\right)\right)\left|\vec{V}_{a}\right|^{2}}$,

where

$\left\langle u_{*}^{2}\right\rangle=N_{i} u_{* i}^{2}+\left(1-N_{i}\right) u_{* o}^{2}$.

In an earlier report (Stössel and Claussen 1992) it was suggested that $\tilde{R} i$ be determined directly from the averaged momentum and turbulent heat fluxes at the blending height. However, that formulation turned out to be inconsistent with the asymptotic limit in which $\tilde{u}_{*}^{2}$ should approach $\left\langle u_{* *}^{2}\right\rangle$ when $\tilde{z}_{0}$ is close to the local roughness lengths, i.e. when surface obstacles become negligible.

\section{Results}

\subsection{Major impact}

The major impact with these modifications of the ASL parameterization for the dynamic forcing of the sea-ice - oceanic mixed-layer model (Stössel 1992) is expected to occur over sea ice with ice compactness between 90 and $99 \%$. This happens to be the dominant type of Antarctic sea-ice distribution in autumn and winter (April to October). Thus, the analysis will focus on winter situations, either on a daily basis (August 28, 1986) or on the seasonal mean (June, July and August 1986). The impact of the new parameterization will be represented largely as differences to the earlier results.
Compared to the earlier simulations with ASL parameterization (Stössel 1992), the winter ice compactness is rather similar, with a tendency toward slightly reduced ice concentrations in the interior ice pack and toward a higher zonal variability of the ice edge. Otherwise, the results in terms of ice extent and width of the marginal ice zone (MIZ) are almost identical.

The most crucial variable indicating the skill of seaice simulations is the ice-thickness distribution. The impact of the new ASL parameterization on that quantity is shown in Fig. 1 as seasonal (quarterly) mean and as difference to the earlier results with the local ASL formulation. Since ice thickness is largely inert to daily fluctuations (in contrast to the ice velocity), its daily distribution pattern is similar to the monthly or seasonal-mean pattern. Generally, the ice-thickness distribution encounters a more dynamic pattern than with the earlier formulation in the sense that ice thicknesses are increased in convergent regions (by up to one metre) and decreased in divergent regions (by up to half a metre). The more dynamic character regionally leads to closer agreement with observations, as far as they are available in terms of ice thickness (Wadhams et al. 1987; Lange and Eicken 1991; Gow et al. 1987).

As a consequence of using the large-scale momentum flux instead of the local one, ice velocities encounter regionally an increase of up to $2 \mathrm{~cm} / \mathrm{s}$ on a seasonal-mean basis (Fig. 2) and of up to $14 \mathrm{~cm} / \mathrm{s}$ on a daily basis (Fig. 3). In regions of the daily convergent ice drift, e.g. in the eastern Ross Sea, ice velocities are

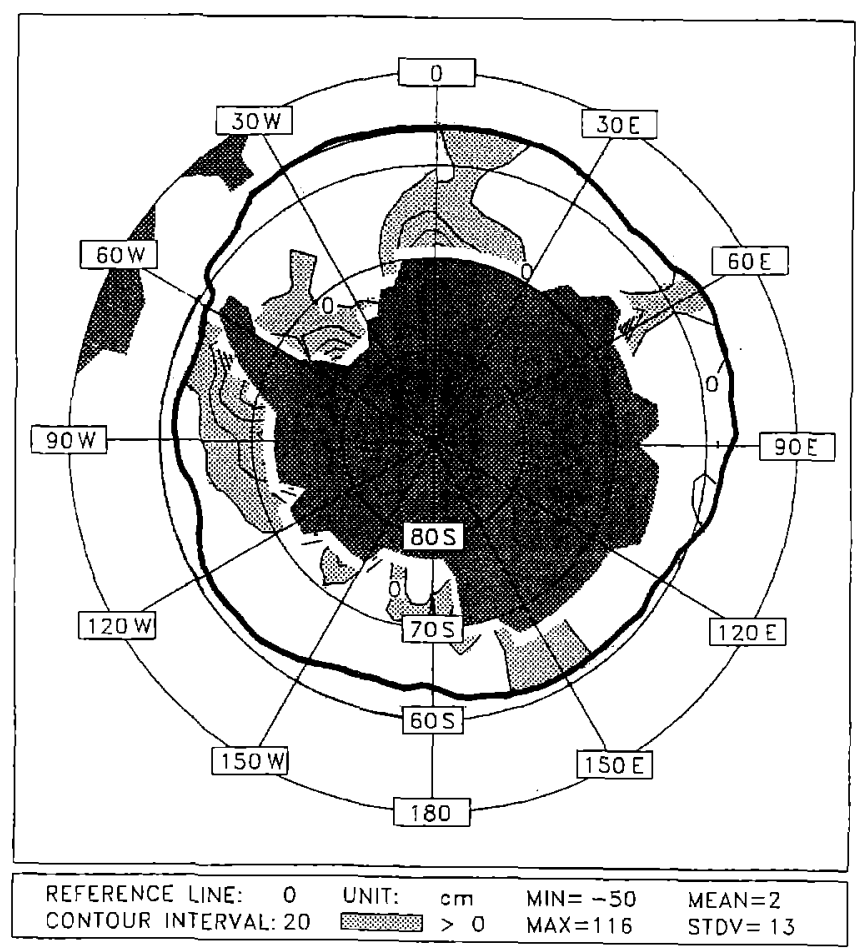

Fig. 1. Quarterly mean (June, July and August, 1986) ice-thickness differences with the dynamic forcing derived from largescale minus local ASL-quantities. The thick solid line represents the contour line of $20 \%$ ice compactness, which is considered to be the ice edge 


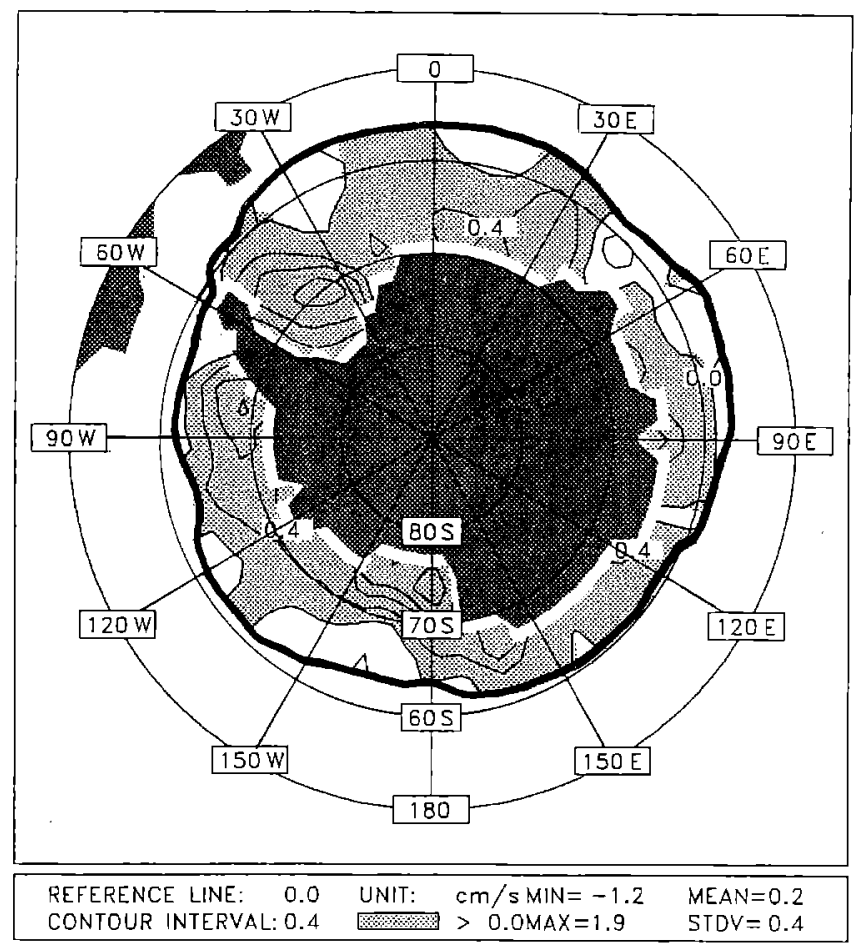

Fig. 2. Ice-velocity differences; otherwise as Fig. 1

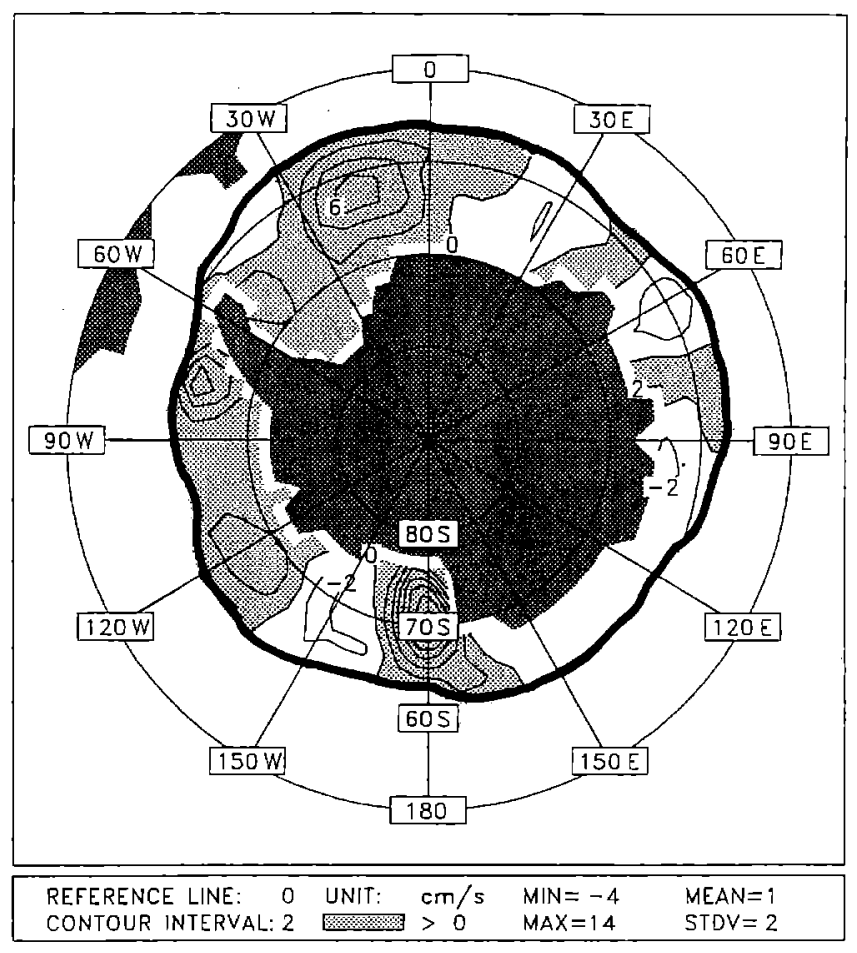

Fig. 3. Daily (August 28, 1986) ice-velocity differences; otherwise as Fig. 1

locally reduced by almost $4 \mathrm{~cm} / \mathrm{s}$. Generally, all velocities in the interior ice pack are increased, with the highest values occurring in the divergent regions of the Weddell and Ross Seas.

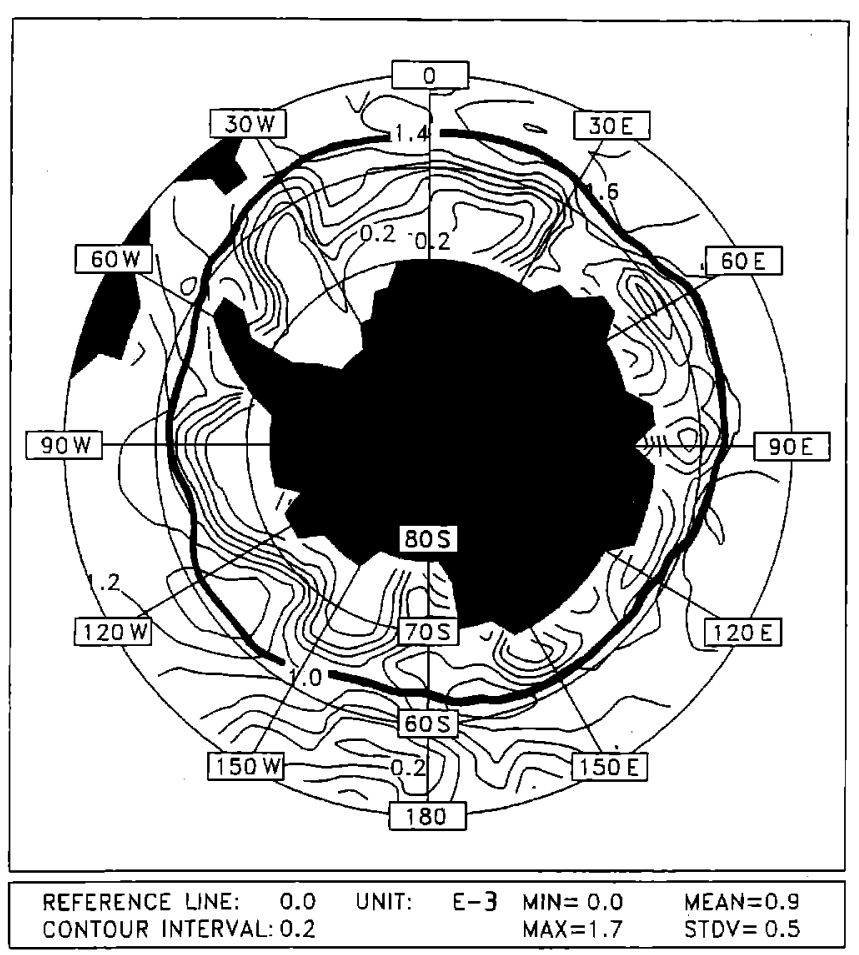

Fig. 4. Daily large-scale drag coefficient

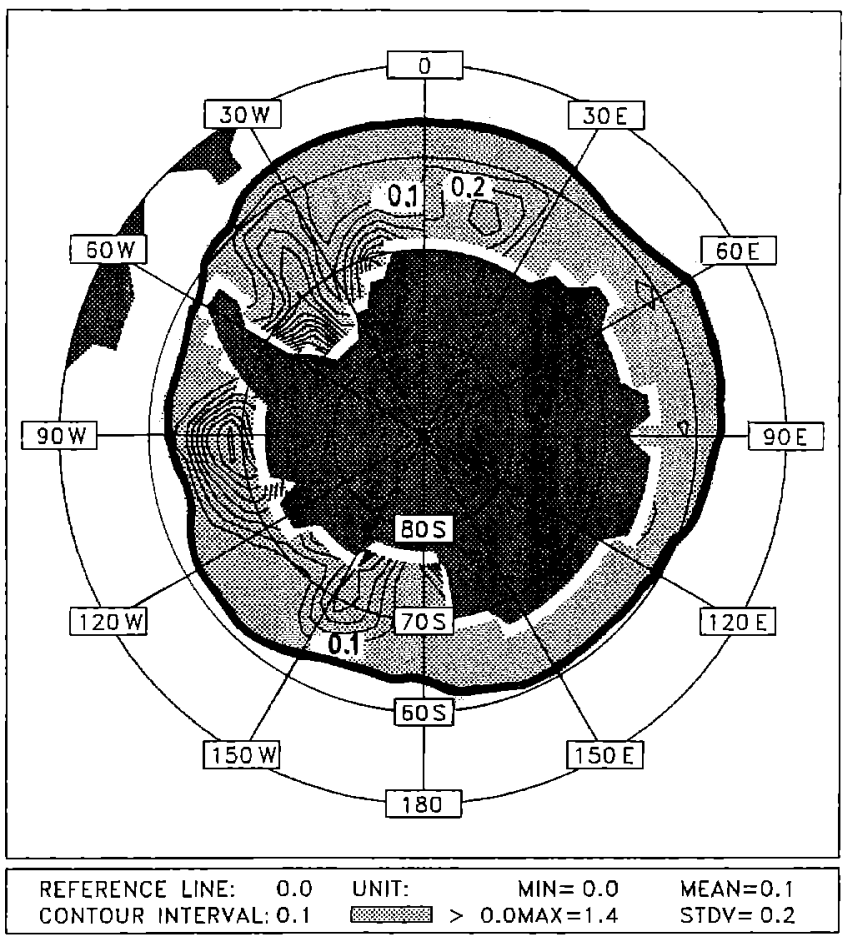

Fig. 5. Daily large-scale Richardson number

Due to the decrease of the ice thickness in divergent ice-drift areas (essentially in the offshore ice-drift region of the Ross Sea), the possibility for new-ice growth is enhanced, leading to more brine rejection followed by a stronger oceanic convection and a subse- 


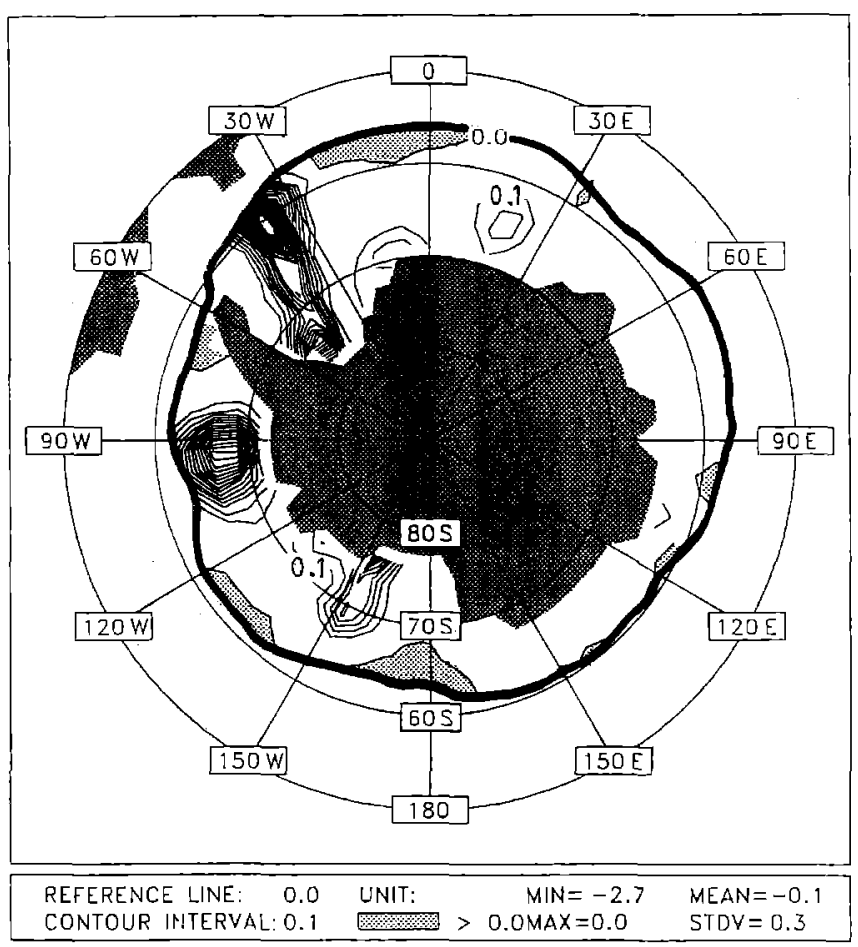

Fig. 6. Daily large-scale minus local Richardson number

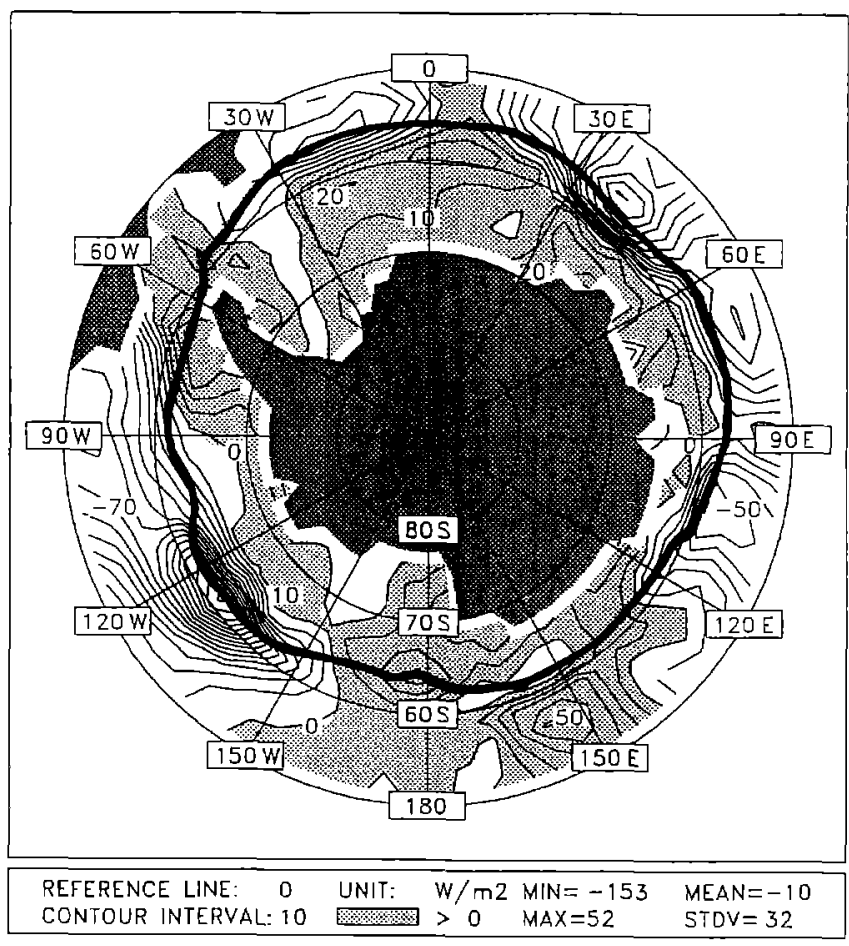

Fig. 7. Daily average sensible heat flux, shaded areas showing downward (positive) fluxes

quent deepening of the oceanic mixed layer (not shown). Accordingly, the wintertime spatially-averaged vertical oceanic (entrainment) heat flux is slightly increased, again leading to closer agreement with ob- servations (Martinson 1990; Gordon and Huber 1990).

\subsection{Analysis}

The reasons for these results are analyzed in the following. The final variable modifying the dynamic forcing of the sea-ice model is the air-ice drag coefficient, which is determined in the present formulation by the large-scale roughness length and the large-scale stability of the ASL. Since it is the accumulated effect of the instantaneous (daily) drag coefficient which determines the characteristic of the seasonal ice field, and not the seasonal mean of the drag coefficient, this is represented here as a daily result. The spatial distribution of its absolute value is shown in Fig. 4. It reflects essentially the synoptic scale variability. Additionally, it shows large areas with low drag in the central and southern Weddell Sea, the Bellingshausen Sea and the eastern Ross Sea. These areas coincide with the patterns of higher static stability in the ASL, presented in Fig. 5 for the same date.

Comparing these results with those from the local momentum calculation (Stössel 1992), there is nevertheless a significant reduction of the stability in those areas, as is demonstrated in Fig. 6. This leads to an increase of the drag coefficient by about $30 \%$. It follows from the large-scale stability function, where instability effects from leads are taken into account.

In this context, it is interesting to note that the averaged turbulent heat fluxes, essentially determined by the sensible heat flux, are negative, i.e. upward, in the areas of $\tilde{R} i$-reduction (Fig. 7). This phenomenon illustrates the Schmidt paradox referred to in Claussen (1991b), demonstrating that while the large-scale stratification is stable, the averaged heat fluxes point upward thus acting 'counter' to the overall vertical gradient of potential temperature.

The other variable modifying the air-ice drag coefficient is the large-scale roughness length, shown in Fig. 8 , which is different to the one used with the local momentum forcing, which was specified to be constant with $z_{0 i}=1 \cdot 10^{-3} \mathrm{~m}$. An overall increase of the roughness length is encountered, which locally (and temporarily) may reach a factor of three.

Since the most cumbersome quantity in the present scheme is the fetch over the ice floes and the leads, it is worthwhile investigating the sensitivity of the largescale roughness length to variations of those quantities. This is done by specifying constant values for $L_{i}$, ranging from $20 \mathrm{~m}$ to $2000 \mathrm{~m}$. The most prominent differences between the upper and the lower case occur in terms of the large-scale roughness length $\left(\tilde{z}_{0}\right)$ and, of course, the blending height $\left(z_{b}\right.$; see Eq. $\left.14 \mathrm{~b}\right)$. While $z_{0}$ increases at the MIZ at the lower margin of $L_{i}$ when compared with the standard prescription (not shown), it adopts almost the local value $z_{0 i}$ at the higher margin. Together with the fact that all other variables, especially, $\tilde{C}_{d}$, do not vary significantly $\left(\tilde{C}_{d}\right.$ at most $10 \%$ ), this implies that the varying large-scale roughness 


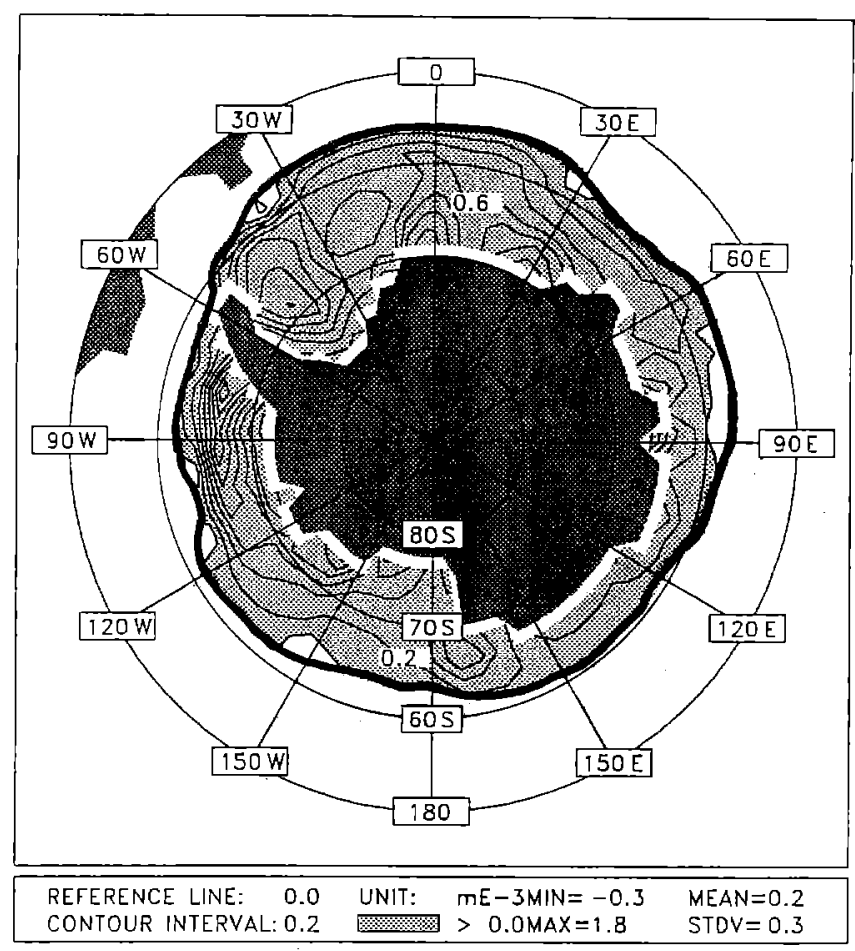

Fig. 8. Daily large-scale minus local roughness length

length has only a small impact on the drag increase, if this is assumed to be determined by the floe size.

More dramatic changes occur when the freeboard of the ice-plus-snow column is increased, e.g. by one metre. Then the large-scale roughness length experiences an increase of up to one order of magnitude, and the large-scale drag coefficient correspondingly by a factor of up to two. Relating such exaggerated freeboards to observed features, one could associate them with heavily ridged ice floes. However, since such features are observed to occur only occasionally in the Southern Ocean region (Lange and Eicken 1991), it is not worthwhile considering them in the present largescale simulation. In the Arctic ice pack, on the other hand, higher pressure ridges and more hummocked ice are observed, which may lead to a stronger contribution to the large-scale roughness length (Overland 1985).

With respect to the differences in stability and roughness length, it is interesting to note that there is a direct interaction between their magnitudes and the dynamics of the ice pack. With the large-scale approach, the ice concentration decreases slightly in most regions, leading to more openings in the ice pack, thus weakening the stratification and increasing the sheltering effects due to floe edges. This represents a positive feedback, which finally leads to an equilibrium state between the large-scale ASL-quantities and the surface characteristics.

\subsection{Assessment}

The question arises immediately, whether the results are reasonable and represent an improvement. Generally, the results are more realistic than the earlier ones in terms of the ice drift and the ice-thickness distribution, judging from the sparse in situ observations available. However, similar results could also be achieved e. g. by changing the empirical parameters of the seaice model or by changing the ice-ocean drag (Stössel et al. 1990; Stössel 1992). Thus, it is necessary to verify the individual ASL quantities.

However, there is an inherent difficulty in deriving large-scale quantities from local observations. HG collected such data from two soundings over the Fram Strait ice pack with an ice compactness of $60 \%$, neutral stratification, a wind velocity of about $5 \mathrm{~m} / \mathrm{s}$, a mean freeboard of $0.4 \mathrm{~m}$ and a mean floe size of $20 \mathrm{~m}$. Apart from the observed large-scale roughness length, which differs by about one order of magnitude from our computations, our results for corresponding situations (except for $L_{i}$ ) are similar. For the large-scale roughness length, the same order of magnitude as measured by HG is achieved when $L_{i}$ is specified as $20 \mathrm{~m}$ and the extended formulation of the skin drag for $\beta<500$ (Eq. 24 in $\mathrm{HG}$ ) is used.

The range of the computed large-scale drag coefficient (see Fig. 4) coincides with corresponding observed values (Andreas et al. 1984; Overland 1985). While $\tilde{C}_{d}$ in Fig. 4 represents primarily the $30 \mathrm{~m}$ value, the one usually referred to in the literature is the $10 \mathrm{~m}$ value, which is generally about $20-30 \%$ higher. The upper and lower values of the large-scale drag coefficient are always correlated with areas of lower and higher stability in the ASL. The minimum of $0.1 \cdot 10^{-3}$ corresponds to a computed stability close to the critical Richardson number of 0.21 , associated with low to moderate winds and very high ice compactnesses. This is within the same order of magnitude as derived from measurements (McBean 1986) and agrees with the empirical derivation of the stable drag coefficient from the neutral one via $z_{a} / L$ (where $L$ denotes the MoninObukhov length) introduced by Banke et al. (1980). Adjusting measurements from low-level flights over the central Arctic in winter (Walter and Overland 1991) to ASL quantities, similar figures can be obtained.

A prominent result is that the large-scale stratification is significantly reduced in areas where the averaged heat flux over the heterogeneous ice pack is upward. It is known that in autumn and wintertime even a small fraction of leads within the ice pack exerts a considerable impact on the overall heat balance (Maykut 1986; Overland and Guest 1991; Worby and Allison 1991). If the leads are favourably distributed, the strong turbulence in the lead area of unstable stratification may act counter to the overall large-scale stratification (Claussen 1991b). It remains to be verified, however, how much this effect contributes to a reduction of the large-scale stability. Recent near-surface $(\approx 30 \mathrm{~m})$ airborne measurements over Artic sea ice in 
autumn (Hartmann et al. 1992), covering a track from ice-free waters via the MIZ to areas of compact sea ice, indicate a high variability of the momentum fluxes. A corresponding variability in the sign of the turbulent heat fluxes suggests that the momentum fluxes are dominated by thermodynamic effects.

A detailed analysis of such data together with their translation to stability in the ASL could give support for the present results. It must be emphasized, however, that a large-scale Richardson number is certainly not a quantity which can directly be derived from measurements. In that sense it should be considered more as an 'artificial' quantity introduced in order to cope with the different scales involved.

\section{Conclusions}

An attempt has been made to modify the dynamic atmospheric forcing for a large-scale sea-ice - oceanic mixed-layer model by deriving large-scale ASL and roughness parameters from local, sub-grid scale quantities. An essential assumption for this derivation is the specification of the forcing at a level of the order of the blending height.

An enhancement of the momentum flux was associated with both a reduction of the ASL-stratification and an increase of the roughness length, as compared to the corresponding values of the earlier local calculations. A significant reduction of the static stability was encountered in regions, where the averaged turbulent heat fluxes pointed upward. The impact on the results of the sea-ice - oceanic mixed-layer model was positive, as far as they could be verified by observations.

The magnitudes of the boundary-layer variables were reasonable, too, noticing that large-scale quantities are difficult to derive from measurements. The large-scale stability and the large-scale roughness length were the key variables in this study. However, recent observations (Overland and Guest 1991; Kottmeier personal communication) suggest that the stratification of the atmospheric boundary layer depends strongly on the radiative budget, being dominated in wintertime by the net longwave radiation, which itself is highly correlated with the presence of clouds. In the present study, clouds are crudely specified by climatological, annual mean and zonally averaged estimates (see Stössel et al. 1990). Further refinements and experiments are clearly needed to analyze their impact.

The present study focused on problems related to the treatment of different relevant scales within a transition zone from one climate component to another, with emphasis on the efforts to couple atmospheric and oceanic models in sea-ice regions. It is suggested that the coupling interface (including sea ice and snow), together with the atmospheric and oceanic boundary layers, should be considered as an integral transition zone between the 'free' media. While the free atmosphere and ocean are characterized mainly by geostrophic balances, the transition zones are dominated by frictional and buoyancy effects, with various interactive exchanges between the different components. If a heterogeneous distribution of two such components is encountered within one model-grid spacing, the interactions are largely determined by local processes. As indicated in this study, it is worthwile incorporating these sub-grid scale processes and to transform them into large-scale quantities in a physically reasonable way in order to provide a proper transition to the "undisturbed' bulk of the atmosphere and the ocean.

Although applied here for forced experiments only, a scheme similar to the one presented is proposed as a tool for matching the GCM-scale coupling over sea-ice covered regions. Together with the implementation of physically more sophisticated sea-ice models in GCMs, this could lead to significant improvements of GCM results in polar regions and lend more credibility to the sensitivity of sea-ice related impacts.

Acknowledgements. We would like to thank K. Herterich for valuable discussions on the concept of sub-grid scale parameterization, K. Hasselmann, L. Bengtsson, P. Lemke and R. Roeckner for valuable comments on the manuscript, M. Grunert and N. Noreiks for finishing the drafts and P. Besemann and S. Beddig for an English review. The Deutsche Wetterdienst (DWD), Offenbach, provided the ECMWF data, which were preprocessed by R. Schnur. This work was mainly sponsored by the Deutsche Forschungsgemeinschaft (DFG) via the Sonderforschungsbereich (SFB) 318 .

\section{References}

Andreas EL, Tucker WB, Ackley SF (1984) Atmospheric boundary-layer modification, drag coefficient, and surface heat flux in the Antarctic marginal ice zone. J Geophys Res 89:649 661

Arya SPS (1975) A drag partition theory for determining the large-scale roughness parameter and wind stress on the Arctic pack ice. J Geophys Res 80:3447-3454

Banke EG, Smith SD, Anderson RJ (1980) Drag coefficients at AIDJEX from sonic anemometer measurements. In: Pritchard RS (ed) Sea ice processes and models. University of Washington Press, Seattle, pp 430-442

Beljaars ACM (1982) The derivation of fluxes from profiles in perturbed areas. Boundary-Layer Meteorol 24:35-55

Beljaars ACM, Holtslag AAM (1991) Flux parameterization over land surfaces for atmospheric models. J Appl Meteorol 30:327-341

Businger JA, Wyngaard JC, Izumi Y, Bradley EF (1971) Flux profile relationships in the atmospheric surface layer. J Atmos Sci 28:181-189

Claussen M (1991a) Local advection processes in the surface layer of the marginal ice zone. Boundary-Layer Meteorol 54:1-27

Claussen M (1991b) Estimation of areally-averaged surface fluxes. Boundary-Layer Meteorol 54:387-410

Claussen M (1992) Scale aggregation in semi-smooth flow. MaxPlanck-Institut für Meteorologie, Report No 87, Hamburg

Cubasch U, Hasselmann K, Höck H, Maier-Reimer E, Mikolajewicz U, Santer BD, Sausen R (1992) Time-dependent greenhouse warming computations with a coupled ocean-atmosphere model. Clim Dyn 8:55-69

Gordon AL, Huber BA (1990) Southern Ocean winter mixed layer. J Geophys Res 95:11655-11672

Gow AJ, Ackley SF, Buck KR, Golden KM (1987) Physical and structural characteristics of Weddell Sea pack ice. US Army, CRREL, Rep. No. 87-14, Hanover, NH 
Hannssen-Bauer I, Gjessing YT (1988) Observations and model calculations of aerodynamic drag on sea ice in the Fram strait. Tellus 40 A:151-161

Hartmann J, Kottmeier C, Wamser C (1992) Radiation and eddy flux experiment 1991 (REFLEX I). Alfred-Wegener-Institut für Polar- und Meeresforschung, Rep on Polar Research 105, Bremerhaven

Lange MA, Eicken H (1991) The sea ice thickness distribution in the northwestern Weddell Sea. J Geophys Res 96:4821-4837

Lemke P, Owens WB, Hibler III WD (1990) A coupled sea ice mixed layer - pycnocline model for the Weddell Sea. J Geophys Res 95:9513-9525

Leppäranta M (1983) A growth model for black ice, snow ice and snow thickness in subarctic basins. Nord Hydrol 14:59-70

Louis JF (1979) A parametric model of vertical eddy fluxes in the atmosphere. Boundary-Layer Meteorol 17:187-202

Manabe S, Spelman MJ, Stouffer RJ (1992) Transient responses of a coupled ocean-atmosphere model to gradual changes of atmospheric $\mathrm{CO}_{2}$. Part II: seasonal response. J Clim 5:105126

Marshall K (1971) Drag measurements in roughness arrays of varying density and distribution. Agr Meteorol 8:269-292

Martinson DG (1990) Evolution of the Southern Ocean winter mixed layer and sea ice: open ocean deepwater formation and ventilation. J Geophys Res 95:11641-11654

Maykut GA (1986) The surface heat and mass balance. NATO ASI Series B 146:395-464

McBean G (1986) The atmospheric boundary layer. NATO ASI Series B 146:283-338

Meehl GA, Washington WM (1990) $\mathrm{CO}_{2}$ climate sensitivity and snow - sea ice albedo parameterization in an atmospheric GCM coupled to a mixed-layer ocean model. Clim Change 6:283-306

Overland JE (1985) Atmospheric boundary layer structure and drag coefficient over sea ice. J Geophys Res 90:9029-9049
Overland JE, Guest PS (1991) The Arctic snow and air temperature budget over sea ice during winter. J Geophys Res $96: 4651-4662$

Overland JE, Davidson KL (1992) Geostrophic drag coefficient over sea ice. Tellus 44A:54-66

Owens WB, Lemke P (1990) Sensitivity studies with a sea ice mixed layer - pycnocline model in the Weddell Sea. J Geophys Res 95:9527-9538

Simmonds I, Budd WF (1991) Sensitivity of the southern hemisphere circulation to leads in the Antarctic pack ice. Q J R Meteorol Soc 117:1003-1024

Stössel A (1985) Thermodynamic calculations of ice production in the northern Baltic proper. Dtsche Hydrogr Z 38:261-284

Stössel A (1992) Sensitivity of Southern Ocean sea-ice simulations to different atmospheric forcing algorithms. Tellus $44 \mathrm{~A}: 395-413$

Stössel A, Claussen M (1992) A new atmospheric surface-layer scheme for a large-scale sea-ice model. Max-Planck-Institut für Meteorologie, Report No 95, Hamburg

Stössel A, Lemke P, Owens WB (1990) Coupled sea ice - mixed layer simulations for the Southern Ocean. J Geophys Res 95:9539-9555

Trenberth KE, Olson JG (1988) ECMWF global analyses 19791986: circulation statistics and data evaluation. NCAR, Technical note TN-300+STR, Boulder, Colorado, USA

Wadhams P, Lange MA, Ackley SF (1987) The ice thickness distribution across the Atlantic sector of the Antarctic Ocean in midwinter. J Geophys Res 92:14535-14552

Walter B, Overland JE (1991) Aircraft observations of the mean and turbulent structure of the atmospheric boundary layer during spring in the Central Arctic. J Geophys Res 96:46634673

Worby AP, Allison I (1991) Ocean-atmosphere energy exchange over thin, variable concentration Antarctic pack ice. Ann Glaciol 15:184-190 